J. Appl. Numer. Optim. 2 (2020), No. 1, pp. 109-120

Available online at http://jano.biemdas.com

https://doi.org/10.23952/jano.2.2020.1.08

\title{
THREE EXTENSIONS OF BUTNARIU-REICH-ZASLAVSKI THEOREM FOR INEXACT INFINITE PRODUCTS OF NONEXPANSIVE MAPPINGS
}

\author{
ALEXANDER J. ZASLAVSKI \\ Department of Mathematics, The Technion - Israel Institute of Technology, 32000 Haifa, Israel
}

\begin{abstract}
In this paper, we study the asymptotic behavior of inexact infinite products of nonexpansive mappings, which take a nonempty, closed subset of a complete metric space into the space under the presence of summable errors, and generalize the known results in the literature for nonexpansive selfmappings on the complete metric space.
\end{abstract}

Keywords. Banach space; Complete metric space; Inexact iteration; Nonexpansive mapping.

\section{INTRODUCTION}

During more than fifty-five years now, there has been a lot of activity regarding the fixed point theory of nonexpansive (that is, 1-Lipschitz) mappings. See, for example, [3, 5, 11, 13, $14,17,18,19,21,22,23,24,25,28,29]$ and the references cited therein. This activity stems from Banach's classical theorem [1] concerning the existence of a unique fixed point for a strict contraction. It also covers the convergence of (inexact) iterates of a nonexpansive mapping to one of its fixed points. Since that seminal result, many developments have taken place in this field including, in particular, studies of feasibility and common fixed point problems, which find important applications in engineering and medical sciences [8, 12, 15, 26, 27, 28, 29].

In [5], it was shown that if any exact orbit of a nonexpansive mapping converges to its fixed point, then this convergence property also holds for its inexact orbits with summable errors. This result was obtained for a self-mapping on a complete metric space $X$. In this paper, we establish variants of this results for inexact infinite products of nonexpansive mappings which take a nonempty, closed subset of a complete metric space $X$ into $X$.

\section{Preliminaries}

Let $(X, \rho)$ be a complete metric space. For every point $z \in X$ and every nonempty set $B \subset X$, let

$$
\rho(z, B)=\inf \{\rho(z, y): y \in B\} .
$$

For every point $z \in X$ and every positive number $\gamma>0$, set

$$
B(z, \gamma)=\{y \in X: \rho(z, y) \leq \gamma\} .
$$

For every mapping $T: X \rightarrow X$, let $T^{0} z=z$ for all $z \in X$.

E-mail address: ajzasl@technion.ac.il.

Received August 17, 2019; Accepted October 27, 2019.

(C)2020 Journal of Applied and Numerical Optimization 
In [5], it was studied the influence of errors on the convergence of orbits of nonexpansive mappings in metric spaces and it was obtained the following result (see also Theorem 2.72 of [25]).

Theorem 2.1. Let $T: X \rightarrow X$ satisfy $\rho(T z, T y) \leq \rho(z, y)$ for all $y, z \in X$, let $F(T)$ be the set of all fixed points of $T$ and let, for each $z \in X$, the sequence $\left\{T^{n} z\right\}_{n=1}^{\infty}$ converge in $(X, \rho)$. Assume that $\left\{z_{n}\right\}_{n=0}^{\infty} \subset X,\left\{\gamma_{n}\right\}_{n=0}^{\infty} \subset(0, \infty)$ satisfies $\sum_{n=0}^{\infty} \gamma_{n}<\infty$ and $\rho\left(z_{n+1}, T z_{n}\right) \leq \gamma_{n}, n=0,1, \ldots$ Then the sequence $\left\{z_{n}\right\}_{n=1}^{\infty}$ converges to a fixed point of $T$ in $(X, \rho)$.

In [21], we obtained the following extension of Theorem 2.1 (see also Theorem 2.74 of [25]).

Theorem 2.2. Let $T: X \rightarrow X$ satisfy $\rho(T z, T y) \leq \rho(z, y)$ for all $z, y \in X$, let $F$ be a nonempty closed subset of $X$ such that, for each $z \in X, \lim _{i \rightarrow \infty} \rho\left(T^{i} z, F\right)=0$. Assume that $\left\{z_{n}\right\}_{n=0}^{\infty} \subset X$, $\left\{\gamma_{n}\right\}_{n=0}^{\infty} \subset(0, \infty)$ satisfies $\sum_{n=0}^{\infty} \gamma_{n}<\infty$ and $\rho\left(z_{n+1}, T z_{n}\right) \leq \gamma_{n}, n=0,1, \ldots$ Then $\lim _{i \rightarrow \infty} \rho\left(z_{n}, F\right)$ $=0$.

In the present paper, we generalize these results for inexact infinite products of nonexpansive mappings, which take a nonempty, closed subset of the complete metric space $X$ into $X$.

Theorem 2.1 finds interesting applications and is an important ingredient in superiorization and perturbation resilience of algorithms. See $[2,4,6,7,9,10,16,20]$ and the references mentioned therein. The superiorization methodology works by taking an iterative algorithm, investigating its perturbation resilience, and then using proactively such perturbations in order to "force" the perturbed algorithm to do in addition to its original task something useful. This methodology can be explained by the following result on convergence of inexact iterates.

Assume that $(X,\|\cdot\|)$ is a Banach space, $\rho(z, y)=\|z-y\|$ for all $z, y \in X$, for each $z \in X$, the sequence $\left\{T^{n} z\right\}_{n=1}^{\infty}$ converges in the norm topology, $z_{0} \in X,\left\{\beta_{k}\right\}_{k=0}^{\infty}$ is a sequence of positive numbers satisfying

$$
\sum_{k=0}^{\infty} \beta_{k}<\infty
$$

$\left\{v_{k}\right\}_{k=0}^{\infty} \subset X$ is a norm bounded sequence and that, for any integer $k \geq 0$,

$$
z_{k+1}=T\left(z_{k}+\beta_{k} v_{k}\right) \text {. }
$$

Then it follows from Theorem 2.1 that the sequence $\left\{z_{k}\right\}_{k=0}^{\infty}$ converges in the norm topology of $X$ and its limit is a fixed point of $T$. In this case, the mapping $A$ is called bounded perturbations resilient (see [6] and Definition 10 of [9]). In other words, if exact iterates of a nonexpansive mapping converge, then its inexact iterates with bounded summable perturbations converge too.

Now, we assume that $z_{0} \in X$ and the sequence $\left\{\beta_{k}\right\}_{k=0}^{\infty}$ satisfying (2.1) are given and we need to find an approximate fixed point of $T$. In order to meet this goal, we construct a sequence $\left\{z_{k}\right\}_{k=1}^{\infty}$ defined by (2.2). Under an appropriate choice of the bounded sequence $\left\{v_{k}\right\}_{k=0}^{\infty}$, the sequence $\left\{z_{k}\right\}_{k=1}^{\infty}$ possesses some useful property. For example, $\left\{f\left(z_{k}\right)\right\}_{k=1}^{\infty}$ can be decreasing, where $f$ is a given function.

\section{MAIN RESUlTS}

3.1. The first result. Let $K$ be a nonempty closed subset of a complete metric space $(X, \rho)$, $F \subset K$ be a nonempty closed set in $(X, \rho)$ and $T_{i}: K \rightarrow X, i=0,1, \ldots$ satisfy for all integers $i \geq 0$,

$$
\rho\left(T_{i} z, T_{i} y\right) \leq \rho(z, y) \text { for all } z, y \in K
$$


We use the following assumptions.

(A) If $z \in K, p \geq 0$ is an integer and the sequence $\left\{\left(\prod_{i=p}^{n} T_{i}\right) z\right\}_{n=p}^{\infty} \subset K$ is well defined, then

$$
\lim _{n \rightarrow \infty} \rho\left(\left(\prod_{i=p}^{n} T_{i}\right) z, F\right)=0 .
$$

We prove the following result.

Theorem 3.1. Let $(A)$ hold, $\tilde{\gamma}>0,\left\{\gamma_{n}\right\}_{n=0}^{\infty} \subset(0, \infty)$ satisfy

$$
\begin{gathered}
\sum_{n=0}^{\infty} \gamma_{n}<\infty, \\
\left\{z_{n}\right\}_{n=0}^{\infty} \subset K, \\
\rho\left(z_{n+1}, T_{n} z_{n}\right) \leq \gamma_{n}, n=0,1, \ldots
\end{gathered}
$$

and

$$
B\left(z_{n}, \tilde{\gamma}\right) \subset K \text { for all sufficiently large natural numbers } n \text {. }
$$

Then

$$
\lim _{i \rightarrow \infty} \rho\left(z_{n}, F\right)=0 .
$$

Proof. By (3.3), there exists an integer $n_{0} \geq 1$ such that

$$
B\left(z_{n}, \tilde{\gamma}\right) \subset K \text { for all integers } n \geq n_{0} .
$$

Let

$$
\varepsilon \in(0, \tilde{\gamma} / 2)
$$

In view of (3.1), we have that there is a natural number

$$
n_{1} \geq n_{0}
$$

such that

$$
\sum_{i=n_{1}}^{\infty} \gamma_{i}<\varepsilon / 4
$$

By induction, we show that, for all integers $n \geq n_{1}$,

$$
\left(\prod_{i=n_{1}}^{n} T_{i}\right) z_{n_{1}} \in K
$$

is well defined and that

$$
\rho\left(\left(\prod_{i=n_{1}}^{n} T_{i}\right) z_{n_{1}}, z_{n+1}\right) \leq \sum_{i=n_{1}}^{n} \gamma_{i} .
$$

Relations (3.2) and (3.4)-(3.7) imply that that

$$
\rho\left(T_{n_{1}} z_{n_{1}}, z_{n_{1}+1}\right) \leq \gamma_{n_{1}}<\tilde{\gamma}
$$

and that

$$
T_{n_{1}} z_{n_{1}} \in B\left(z_{n_{1}+1}, \tilde{\gamma}\right) \subset K
$$

Assume that $n \geq n_{1}$ is an integer,

$$
\left(\prod_{i=n_{1}}^{n} T_{i}\right) z_{n_{1}} \in K
$$


is well defined and that (3.8) holds. (In view of (3.9) and (3.10), our assumption holds for $\left.n=n_{1}\right)$. It follows from (3.2) and (3.8) that

$$
\begin{aligned}
\rho\left(\left(\prod_{i=n_{1}}^{n+1} T_{i}\right) z_{n_{1}}, z_{n+2}\right) & \leq \rho\left(\left(\prod_{i=n_{1}}^{n+1} T_{i}\right) z_{n_{1}}, T_{n+1} z_{n+1}\right)+\rho\left(T_{n+1} z_{n+1}, z_{n+2}\right) \\
& \leq \rho\left(\left(\prod_{i=n_{1}}^{n} T_{i}\right) z_{n_{1}}, z_{n+1}\right)+\gamma_{n+1} \leq \sum_{i=n_{1}}^{n+1} \gamma_{i} .
\end{aligned}
$$

In view of (3.5), (3.7) and (3.11), we have

$$
\rho\left(\left(\prod_{i=n_{1}}^{n+1} T_{i}\right) z_{n_{1}}, z_{n+2}\right) \leq \tilde{\gamma}
$$

By (3.4), (3.6) and (3.12), we have

$$
\left(\prod_{i=n_{1}}^{n+1} T_{i}\right) z_{n_{1}} \in B\left(z_{n+2}, \tilde{\gamma}\right) \subset K .
$$

It follows from the inclusion above and (3.11) that the assumption made for $n$ also holds for $n+1$. Therefore by induction we showed that

$$
\left(\prod_{i=n_{1}}^{n} T_{i}\right) z_{n_{1}} \in K \text { for all integers } n \geq n_{1}
$$

and that (3.8) holds for all integers $n \geq n_{1}$. From our assumptions,

$$
\lim _{n \rightarrow \infty} \rho\left(\left(\prod_{i=n_{1}}^{n} T_{i}\right) z_{n_{1}}, F\right)=0 .
$$

It follows from (3.13) that there exists a natural number $n_{2}>n_{1}$ such that, for all natural numbers $n \geq n_{2}$,

$$
\rho\left(\left(\prod_{i=n_{1}}^{n} T_{i}\right) z_{n_{1}}, F\right)<\varepsilon / 4 .
$$

By (3.7), (3.8) and (3.14), for all integers $n \geq n_{2}$, we have

$$
\begin{aligned}
\rho\left(z_{n+p}, F\right) & \leq \rho\left(z_{n+p},\left(\prod_{i=n_{1}}^{n} T_{i}\right) z_{n_{1}}\right)+\rho\left(\left(\prod_{i=n_{1}}^{n} T_{i}\right) z_{n_{1}}, F\right) \\
& <\varepsilon / 2 .
\end{aligned}
$$

Since $\varepsilon$ is any number belonging to $(0, \tilde{\gamma} / 2)$, we conclude that $\lim _{i \rightarrow \infty} \rho\left(z_{i}, F\right)=0$. This completes the proof of Theorem 3.1

3.2. The second result. Let $X$ be a nonempty closed subset of a Banach space $(E,\|\cdot\|)$ with a dual space $\left(E^{*},\|\cdot\|_{*}\right)$ and let $T: X \rightarrow X$ satisfy $\|T z-T y\| \leq\|z-y\|$ for each $z, y \in X$. As usual, we denote by $T^{0}$ the identity self-mapping of $X$. Consider the following assumptions.

(A1) For each $z \in X$, the sequence $\left\{T^{n} z\right\}_{n=1}^{\infty}$ converges weakly in $X$.

(A2) For each $z \in X$, the sequence $\left\{T^{n} z\right\}_{n=1}^{\infty}$ converges weakly in $X$ to a fixed point of $T$.

The following result was obtained in [5] (see also Theorem 2.73 of [25]). 
Theorem 3.2. Assume that (A1) holds. Let $\left\{\gamma_{n}\right\}_{n=0}^{\infty} \subset(0, \infty), \sum_{n=0}^{\infty} \gamma_{n}<\infty,\left\{z_{n}\right\}_{n=0}^{\infty} \subset X$, and $\left\|z_{n+1}-T z_{n}\right\| \leq \gamma_{n}, n=0,1, \ldots$ Then the sequence $\left\{z_{n}\right\}_{n=1}^{\infty}$ converges weakly in $X$. Moreover, if (A2) holds, then its limit is a fixed point of $T$.

In the present paper, we generalize this result for infinite products of nonexpansive mappings, which take a nonempty, closed subset of the Banach space $E$ into $E$.

Let $K$ be a nonempty closed subset of $E, F$ be a nonempty closed subset of $K$ (in the norm topology) and $T_{i}: K \rightarrow E, i=0,1, \ldots$ satisfy, for all integers $i \geq 0$,

$$
\begin{gathered}
\left\|T_{i} z-T_{i} y\right\| \leq\|z-y\| \text { for each } z, y \in K . \\
T_{i} z=z \text { for all } z \in F .
\end{gathered}
$$

We use following assumptions.

(A3) If $z \in K, p \geq 0$ is an integer and the sequence $\left\{\left(\prod_{i=p}^{n} T_{i}\right) z\right\}_{n=p}^{\infty} \subset K$ is well defined, then it converges weakly in $E$.

(A4) If $z \in K, p \geq 0$ is an integer and the sequence $\left\{\left(\prod_{i=p}^{n} T_{i}\right) z\right\}_{n=p}^{\infty} \subset K$ is well defined, then it converges weakly in $E$ to a point of $F$.

We prove the following result.

Theorem 3.3. Let (A3) hold, $\left\{z_{n}\right\}_{n=0}^{\infty} \subset K, \tilde{\gamma}>0$, and $\left\{\gamma_{n}\right\}_{n=0}^{\infty} \subset(0, \infty)$ satisfy

$$
\begin{gathered}
\sum_{n=0}^{\infty} \gamma_{n}<\infty, \\
\left\|z_{n+1}-T_{n} z_{n}\right\| \leq \gamma_{n}, n=0,1, \ldots .
\end{gathered}
$$

Let

$$
B\left(z_{n}, \tilde{\gamma}\right) \subset K \text { for all sufficiently large natural numbers } n \text {. }
$$

Then the sequence $\left\{z_{n}\right\}_{n=1}^{\infty}$ converges weakly in $E$ and if $K$ is weakly closed, then the limit belongs to K. Moreover, if (A4) holds, then the limit belongs to F.

Proof. By (3.17), there exists an integer $n_{0} \geq 1$ such that

$$
B\left(z_{n}, \tilde{\gamma}\right) \subset K \text { for all integers } n \geq n_{0} .
$$

In view of (3.15), we have that there is a natural number $n_{1}$ such that

$$
n_{1}>n_{0} \text { and } \sum_{i=n_{1}}^{\infty} \gamma_{i}<\tilde{\gamma} / 4 \text {. }
$$

Let

$$
p \geq n_{1}
$$

be an integer. By induction, we show that, for all integers $n \geq p$,

$$
\left(\prod_{i=p}^{n} T_{i}\right) z_{p} \in K
$$

is well defined. Relations (3.16) and (3.18)-(3.20) imply that that $B\left(z_{p+1}, \tilde{\gamma}\right) \subset K$ and

$$
T_{p} z_{p} \in K,\left\|T_{p} z_{p}-z_{p+1}\right\| \leq \gamma_{p} .
$$


Assume that $n \geq 0$ is an integer,

$$
\left(\prod_{i=p}^{n+p} T_{i}\right) z_{p} \in K
$$

is well defined and that

$$
\left\|\left(\prod_{i=p}^{p+n} T_{i}\right) z_{p}-z_{n+1+p}\right\| \leq \sum_{i=p}^{n+p} \gamma_{i} .
$$

(In view of (3.21), our assumption holds for $n=0$ ). By (3.22), we have that

$$
\left(\prod_{i=p}^{p+n+1} T_{i}\right) z_{p} \in E
$$

is well defined. Relations (3.18), (3.19) and (3.20) imply that $B\left(z_{n+p+1}, \tilde{\gamma}\right) \subset K$. It follows from (3.16) and (3.23) that

$$
\begin{aligned}
& \left\|\left(\prod_{i=p}^{n+1+p} T_{i}\right) z_{p}-z_{n+2+p}\right\| \\
& \leq\left\|\left(\prod_{i=p}^{n+1+p} T_{i}\right) z_{p}-T_{n+1+p} z_{n+1+p}\right\|+\left\|T_{n+1+p} z_{n+1+p}-z_{n+2+p}\right\| \\
& \leq\left\|\left(\prod_{i=p}^{n+p} T_{i}\right) z_{p}-z_{n+1+p}\right\|+\gamma_{n+1+p} \\
& \leq \sum_{i=p}^{n+p+1} \gamma_{i} .
\end{aligned}
$$

In view of (3.19), (3.20) and (3.24), we have

$$
\left\|\left(\prod_{i=p}^{p+n+1} T_{i}\right) z_{p}-z_{n+2+p}\right\| \leq \tilde{\gamma} / 4 .
$$

By (3.18), (3.20) and (3.25), we have

$$
\left(\prod_{i=p}^{n+1+p} T_{i}\right) z_{p} \in K
$$

It follows from (3.24) and (3.26) that the assumption made for $n$ also holds for $n+1$. Therefore by induction we showed that (3.22) and (3.23) hold for all integers $n \geq 0$. Assumption (A3) implies that the sequence $\left\{\left(\prod_{i=p}^{n+p} T_{i}\right) z_{p}\right\}_{n=0}^{\infty}$ converges weakly to $y_{p} \in E$. If the set $K$ is weakly closed, then

$$
y_{p} \in K
$$

and if (A4) holds, then $y_{p} \in F$. Fix an integer $q \geq 0$. By using (3.23), we have

$$
\left\|\left(\prod_{j=p}^{q+p} T_{j}\right) z_{p}-z_{q+p+1}\right\| \leq \sum_{j=p}^{\infty} \gamma_{j} .
$$


It follows from that, for each integer $i \geq 1$,

$$
\begin{aligned}
\left\|\left(\prod_{j=p}^{q+p+i} T_{j}\right) z_{p}-\left(\prod_{j=p+q+1}^{q+p+i} T_{j}\right) z_{q+p+1}\right\| & \leq\left\|\left(\prod_{j=p}^{q+p} T_{j}\right) z_{p}-z_{q+p+1}\right\| \\
& \leq \sum_{j=p}^{\infty} \gamma_{j} .
\end{aligned}
$$

The equations

$$
\begin{gathered}
y_{p}=\lim _{n \rightarrow \infty}\left(\prod_{j=p}^{n} T_{j}\right) z_{p} \text { in the weak topology, } \\
y_{q+p+1}=\lim _{n \rightarrow \infty}\left(\prod_{j=p+q+1}^{n} T_{j}\right) z_{q+p+1} \text { in the weak topology }
\end{gathered}
$$

hold. By (3.28), (3.29) and (3.30), for each $f \in E^{*}$ satisfying $\|f\|_{*} \leq 1$, we have

$$
\begin{aligned}
\left|f\left(y_{q}\right)-f\left(y_{q+p+1}\right)\right| & =\lim _{n \rightarrow \infty}\left|f\left(\left(\prod_{j=p}^{n} T_{j}\right) z_{p}\right)-f\left(\left(\prod_{j=p+q+1}^{n} T_{j}\right) z_{p+q+1}\right)\right| \\
& \leq \limsup _{n \rightarrow \infty}\|f\|\left\|\left(\prod_{j=p}^{n} T_{j}\right) z_{p}-\left(\prod_{j=p+q+1}^{n} T_{j}\right) z_{p+q+1}\right\| \\
& \leq \sum_{j=p}^{\infty} \gamma_{j} .
\end{aligned}
$$

This implies that

$$
\left\|y_{p}-y_{p+q+1}\right\| \leq \sum_{j=p}^{\infty} \gamma_{j} .
$$

Since the above inequality holds for each integer $p \geq n_{1}$ and each integer $q \geq 0$, and since $\sum_{j=0}^{\infty} \gamma_{j}<\infty$, we conclude that $\left\{y_{k}\right\}_{k=1}^{\infty}$ is a Cauchy sequence and there exists

$$
y_{*}=\lim _{k \rightarrow \infty} y_{k}
$$

in the norm topology of $E$. Clearly, if $K$ is weakly closed, then $y_{*} \in K$ and if (A4) holds, then $y_{*} \in F$ By (3.32) and (3.33), we have

$$
\left\|y_{p}-y_{*}\right\| \leq \sum_{j=p}^{\infty} \gamma_{j}
$$

for all integers $p \geq n_{1}$.

In order to complete the proof, it is sufficient to show that $\lim _{k \rightarrow \infty} z_{k}=y_{*}$ in the weak topology. Let $f \in E^{*}$ be a continuous linear functional on $E$ such that $\|f\|_{*} \leq 1$ and let $\varepsilon>0$ be given. It is sufficient to show that $\left|f\left(y_{*}-z_{i}\right)\right| \leq \varepsilon$ for all large enough integers $i \geq 0$. In view of (3.15), there is an integer $p \geq n_{1}$ such that

$$
\sum_{j=p}^{\infty} \gamma_{j}<\varepsilon / 4
$$


It follows from (3.27) and (3.34) that, for each integer $n \geq 0$,

$$
\begin{aligned}
\left|f\left(y_{*}-z_{p+n+1}\right)\right| & \leq\left|f\left(y_{*}-y_{p}\right)\right|+\left|f\left(y_{p}-\left(\prod_{j=p}^{n+p} T_{j}\right) z_{p}\right)\right|+\left|f\left(\left(\prod_{j=p}^{n+p} T_{j}\right) z_{p}-z_{n+p+1}\right)\right| \\
& \leq\left\|y_{*}-y_{p}\right\|+\left|f\left(y_{p}-\left(\prod_{j=p}^{n+p} T_{j}\right) z_{p}\right)\right|+\left\|\left(\prod_{j=p}^{n+p} T_{j}\right) z_{p}-z_{n+p+1}\right\| \\
& \leq \sum_{j=p}^{\infty} \gamma_{j}+\left|f\left(y_{p}-\left(\prod_{j=p}^{n+p} T_{j}\right) z_{p}\right)\right|+\sum_{j=p}^{\infty} \gamma_{j} .
\end{aligned}
$$

By (3.29), there is a natural number $m_{0}$ such that

$$
\left|f\left(y_{p}-\left(\prod_{j=p}^{n+p} T_{j}\right) z_{p}\right)\right|<\varepsilon / 4 \text { for all natural numbers } n \geq m_{0} .
$$

It follows from (3.35) that, for each integer $n \geq m_{0}$,

$$
\left|f\left(y_{*}-z_{p+n+1}\right)\right| \leq \varepsilon / 4+\varepsilon / 4+\varepsilon / 4=3 \varepsilon / 4 .
$$

Theorem 3.3 is proved.

3.3. The third result. Let $(E,\|\cdot\|)$ be a reflexive Banach space with a dual space $\left(E^{*},\|\cdot\|_{*}\right)$.

In [21], we obtained the following result (see also Theorem 2.75 of [25]).

Theorem 3.4. Let $X$ be a nonempty closed subset of $E$, and let $T: X \rightarrow X$ satisfy $\|T z-T y\| \leq$ $\|z-y\|$ for all $z, y \in X$. Let $F$ be a nonempty closed subset of $X$ such that, for each $z \in X$, the sequence $\left\{T^{n} z\right\}_{n=1}^{\infty}$ is bounded and all its weak limit points belong to $F$. Assume that $\left\{z_{n}\right\}_{n=0}^{\infty} \subset X,\left\{\gamma_{n}\right\}_{n=0}^{\infty} \subset(0, \infty)$ satisfies $\sum_{n=0}^{\infty} \gamma_{n}<\infty$ and $\left\|z_{n+1}-T z_{n}\right\| \leq \gamma_{n}, n=0,1, \ldots$ Then the sequence $\left\{z_{n}\right\}_{n=0}^{\infty}$ is bounded and all its weak limit points belong to $F$.

In the present paper, we generalize this result for infinite products of nonexpansive mappings which take a nonempty, closed subset of the Banach space $E$ into $E$.

Let $K$ be a nonempty closed subset of $E$, (in the norm topology) and let $T_{i}: K \rightarrow E, i=0,1, \ldots$ satisfy, for all integers $i \geq 0$,

$$
\left\|T_{i} z-T_{i} y\right\| \leq\|z-y\| \text { for each } z, y \in K .
$$

We prove the following result.

Theorem 3.5. Let $F \subset K$ be a nonempty closed in the norm topology set and let the following property holds:

(a) if $z \in K, p \geq 0$ is an integer and the sequence $\left\{\left(\prod_{i=p}^{n} T_{i}\right) z\right\}_{n=p}^{\infty} \subset K$ is well defined, then it is bounded and all its weak limit points belongs to $F$.

Assume that $\left\{\gamma_{n}\right\}_{n=0}^{\infty} \subset(0, \infty)$ satisfies

$$
\begin{gathered}
\sum_{n=0}^{\infty} \gamma_{n}<\infty, \\
\left\{z_{n}\right\}_{n=0}^{\infty} \subset K, \\
\left\|z_{n+1}-T_{n} z_{n}\right\| \leq \gamma_{n}, n=0,1, \ldots \\
\tilde{\gamma}>0
\end{gathered}
$$


and

$$
B\left(z_{n}, \tilde{\gamma}\right) \subset K \text { for all sufficiently large natural numbers } n \text {. }
$$

Then the sequence $\left\{z_{n}\right\}_{n=1}^{\infty}$ is bounded and all its weak limit points belongs to $F$.

Proof. By (3.39), there exists an integer $n_{0} \geq 1$ such that

$$
B\left(z_{n}, \tilde{\gamma}\right) \subset K \text { for all integers } n \geq n_{0} .
$$

Let

$$
\varepsilon \in(0, \tilde{\gamma} / 4)
$$

In view of (3.37), there is a natural number

$$
n_{1} \geq n_{0}
$$

such that

$$
\sum_{i=n_{1}}^{\infty} \gamma_{i}<\varepsilon / 4
$$

Let

$$
p \geq n_{1}
$$

be an integer. Relations (3.42) and (3.44) imply that

$$
B\left(z_{p+1}, \tilde{\gamma}\right) \subset K
$$

By (3.38), (3.41), (3.43), (3.44) and (3.45), we have

$$
T_{p} z_{p} \in B\left(z_{p+1}, \tilde{\gamma}\right) \subset K
$$

and

$$
\left\|T_{p} z_{p}-z_{p+1}\right\| \leq \gamma_{p}
$$

By induction, we show that, for all integer $n \geq p$,

$$
\left(\prod_{i=p}^{n} T_{i}\right) z_{p} \in K
$$

is well defined. Assume that $n \geq p$ is an integer,

$$
\left(\prod_{i=p}^{n} T_{i}\right) z_{p} \in K
$$

is well defined and that

$$
\left\|\left(\prod_{i=p}^{n} T_{i}\right) z_{p}-z_{n+1}\right\| \leq \sum_{i=p}^{n} \gamma_{i} .
$$

(In view of (3.46) and (3.47), our assumption holds for $n=p$ ). By (3.48), we have

$$
\left(\prod_{i=p}^{n+1} T_{i}\right) z_{p} \in E
$$


is well defined. Relations (3.40), (3.42) and (3.44) imply that $B\left(z_{n+1}, \tilde{\gamma}\right) \subset K$. It follows from (3.36), (3.38) and (3.49) that

$$
\begin{aligned}
\left\|\left(\prod_{i=p}^{n+1} T_{i}\right) z_{p}-z_{n+2}\right\| & \leq\left\|\left(\prod_{i=p}^{n+1} T_{i}\right) z_{p}-T_{n+1} z_{n+1}\right\|+\left\|T_{n+1} z_{n+1}-z_{n+2}\right\| \\
& \leq\left\|\left(\prod_{i=p}^{n} T_{i}\right) z_{p}-z_{n+1}\right\|+\gamma_{n+1} \\
& \leq \sum_{i=p}^{n+1} \gamma_{i} .
\end{aligned}
$$

In view of (3.41), (3.43) and (3.44), we have

$$
\left\|\left(\prod_{i=p}^{n+1} T_{i}\right) z_{p}-z_{n+2}\right\| \leq \tilde{\gamma} / 4
$$

which together with (3.40) implies that $\left(\prod_{i=p}^{n+1} T_{i}\right) z_{p} \in K$ and the assumption made for $n$ also holds for $n+1$. Therefore by induction we showed that (3.48) and (3.49) hold for all integers $n \geq p$.

Property (a) implies that the sequence $\left\{\left(\prod_{i=p}^{n} T_{i}\right) z_{p}\right\}_{n=p}^{\infty}$ is bounded and all its weak limit points belong to $F$. Together with (3.49) this implies that sequence $\left\{z_{n}\right\}_{n=0}^{\infty}$ is bounded.

Let $\xi$ be a weakly limit point of $\left\{z_{n}\right\}_{n=0}^{\infty}$. There exists a subsequence $\left\{z_{i_{n}}\right\}_{n=1}^{\infty}$ which converges weakly to $\xi \in E$. We may assume without loss of generality that

$$
\left\{\left(\prod_{j=p}^{i_{n}-1} T_{j}\right) z_{p}\right\}_{n=1}^{\infty}
$$

weakly converges to $\tilde{\xi} \in F$.

From (3.43), (3.44) and (3.49), for each $f \in E^{*}$ satisfying $\|f\|_{*} \leq 1$, we have

$$
\begin{aligned}
|f(\xi)-f(\tilde{\xi})| & =\lim _{n \rightarrow \infty}\left|f\left(z_{i_{n}}\right)-f\left(\left(\prod_{j=p}^{i_{n}-1} T_{j}\right) z_{p}\right)\right| \\
& \leq \limsup _{n \rightarrow \infty}\|f\|\left\|z_{i_{n}}-\left(\prod_{j=p}^{i_{n}-1} T_{j}\right) z_{p}\right\| \\
& \leq \sum_{j=p}^{\infty} \gamma_{j} \\
& <\varepsilon / 4 .
\end{aligned}
$$

This implies that $\|\xi-\tilde{\xi}\| \leq \varepsilon$. Since $\tilde{\xi} \in F$, we have $\rho(z, F) \leq \varepsilon$. Since $\varepsilon$ is any number satisfying (3.41), we conclude that $\xi \in F$. Theorem 3.5 is proved.

It should be mentioned that in our proofs assumptions (A), (A3), (A4), (a), (3.3), (3.17) and (3.39) play a crucial role. Mappings for which assumption (A) holds are considered in $[25,28]$. Assumptions (A3), (A4) and (a) usually hold if the set $K$ is bounded and the space $X$ is Hilbert. Assumptions (3.3), (3.17) and (3.39) for a given sequence $\left\{z_{k}\right\}_{k=0}^{\infty}$ can be checked 
numerically. They mean that the distance of every element of the sequence from the boundary of $K$ is bounded from below by a positive constant.

\section{REFERENCES}

[1] S. Banach, Sur les opérations dans les ensembles abstraits et leur application aux équations intégrales, Fund. Math. 3 (1922), 133-181.

[2] H. H. Bauschke, V. R. Koch, Projection methods: Swiss army knives for solving feasibility and best approximation problems with half-spaces, Contemporary Math. 636 (2015), 1-40.

[3] A. Betiuk-Pilarska, T. Domínguez Benavides, Fixed points for nonexpansive mappings and generalized nonexpansive mappings on Banach lattices, Pure Appl. Func. Anal. 1 (2016), 343-359.

[4] D. Butnariu, R. Davidi, G. T. Herman, I. G. Kazantsev, Stable convergence behavior under summable perturbations of a class of projection methods for convex feasibility and optimization problems, IEEE J. Sel. Topics Signal Processing 1 (2007), 540-547.

[5] D. Butnariu, S. Reich, A. J. Zaslavski, Convergence to fixed points of inexact orbits of Bregman-monotone and of nonexpansive operators in Banach spaces, Fixed Point Theory and its Applications, pp. 11-32, Yokohama Publishers, Yokohama, 2006

[6] Y. Censor, R. Davidi, G. T. Herman, Perturbation resilience and superiorization of iterative algorithms, Inverse Probl. 26 (2010), 065008.

[7] Y. Censor, R. Davidi, G. T. Herman, R. W. Schulte, L. Tetruashvili, Projected subgradient minimization versus superiorization, J. Optim. Theory Appl. 160 (2014), 730-747.

[8] Y. Censor, M. Zaknoon, Algorithms and convergence results of projection methods for inconsistent feasibility problems: a review, Pure Appl. Func. Anal. 3 (2018), 565-586.

[9] Y. Censor, A. J. Zaslavski, Convergence and perturbation resilience of dynamic string-averaging projection methods, Comput. Optim. Appl. 54 (2013), 65-76.

[10] Y. Censor, A. J. Zaslavski, Strict Fejer monotonicity by superiorization of feasibility-seeking projection methods, J. Optim. Theory Appl. 165 (2015), 172-187.

[11] F. S. de Blasi, J. Myjak, S. Reich, A. J. Zaslavski, Generic existence and approximation of fixed points for nonexpansive set-valued maps, Set-Valued Var. Anal. 17 (2009), 97-112.

[12] A. Gibali, A new split inverse problem and an application to least intensity feasible solutions, Pure Appl. Funct. Anal. 2 (2017), 243-258.

[13] K. Goebel, W. A. Kirk, Topics in Metric Fixed Point Theory, Cambridge University Press, Cambridge, 1990.

[14] K. Goebel, S. Reich, Uniform Convexity, Hyperbolic Geometry, and Nonexpansive Mappings, Marcel Dekker, New York and Basel, 1984.

[15] L. G. Gurin, B. T. Poljak, E. V. Raik, ' Projection methods for finding a common point of convex sets, Zhurnal Vychislitel'noi Matematiki i Matematicheskoi Fiziki 7 (1967), 1211-1228.

[16] G. T. Herman, R. Davidi, Image reconstruction from a small number of projections, Inverse Probl. 2 (2008), $1-17$.

[17] J. Jachymski, Extensions of the Dugundji-Granas and Nadler's theorems on the continuity of fixed points, Pure Appl. Funct. Anal. 2 (2017), 657-666.

[18] W. A. Kirk, Contraction Mappings and Extensions, Handbook of Metric Fixed Point Theory, pp. 1-34, Kluwer, Dordrecht, 2001.

[19] R. Kubota, W. Takahashi, Y. Takeuchi, Extensions of Browder's demiclosedness principle and Reich's lemma and their applications, Pure Appl. Func. Anal. 1 (2016), 63-84.

[20] T. Nikazad, R. Davidi, G. T. Herman, Accelerated perturbation-resilient block-iterative projection methods with application to image reconstruction, Inverse Probl. 28 (2012), 19 pp.

[21] E. Pustylnyk, S. Reich, A. J. Zaslavski, Inexact orbits of nonexpansive mappings, Taiwanese J. Math. 12 (2008), 1511-1523.

[22] S. Reich, A. J. Zaslavski, Well-posedness of fixed point problems, Far East J. Math. Sci. Special Volume (Functional Analysis and Its Applications), Part III (2001), 393-401.

[23] S. Reich, A. J. Zaslavski, Generic Aspects of Metric Fixed Point Theory, Handbook of Metric Fixed Point Theory, PP. 557-575, Kluwer, Dordrecht, 2001. 
[24] S. Reich, A. J. Zaslavski, Convergence to attractors under perturbations, Commun. Math. Anal. 10 (2011), 57-63.

[25] S. Reich, A. J. Zaslavski, Genericity in Nonlinear Analysis, Springer, New York, 2014.

[26] W. Takahashi, The split common fixed point problem and the shrinking projection method for new nonlinear mappings in two Banach spaces, Pure Applied Funct. Anal. 2 (2017), 685-699.

[27] W. Takahashi, A general iterative method for split common fixed point problems in Hilbert spaces and applications, Pure Appl. Funct. Anal. 3 (2018), 349-369.

[28] A. J. Zaslavski, Approximate solutions of common fixed point problems, Springer Optimization and Its Applications, Springer, Cham, 2016.

[29] A. J. Zaslavski, Algorithms for solving common fixed point problems, Springer Optimization and Its Applications, Springer, Cham, 2018. 